
Kidney
Blood Pressure
Research

Kidney Blood Press Res 2015;40:188-199

\title{
Glomerular Damage in Experimental Proliferative Glomerulonephritis Under Glomerular Capillary Hypertension
}

\author{
Pei-Rong Wang ${ }^{a} \quad$ Hiroshi Kitamura ${ }^{b} \quad$ Akira Shimizuc $^{c} \quad$ Nobuaki Yamanakad \\ aDepartment of Pediatrics, Second Hospital of Shandong University, Jinan, PR China; ${ }^{b}$ Renal \\ Pathology Section, National Hospital Organization Chiba-East Hospital, Chiba; 'Department of \\ Analytic Human Pathology, Nippon Medical School; ' $T o k y o$ Kidney Research Institute, Tokyo, Japan
}

\section{Key Words}

SHR-SP • Unilateral nephrectomy • Thy-1 nephritis • Hypertension • Glomerular damage

\begin{abstract}
Background/Aims: Immunologically and hemodynamically mediated the destruction of glomerular architecture is thought to be the major causes of end-stage renal failure. The purpose of this study is to evaluate the effect of glomerular hypertension on glomerular injury and the progression of glomerular sclerosis after Thy-1 nephritis was induced. Method: Thy-1 nephritis was induced in the stroke-prone spontaneously hypertensive rat strain (SHR-SP) (group SP) and in age-matched Wistar-Kyoto (WKY) (group WKY) rats, following unilateral nephrectomy (UNX), and a vehicle was injected alone in UNX SHR-SP as control (group SC). Result: The degree of glomerular damage in response to a single dose of antithy- 1 antibody, and its functional consequences (eg. proteinuria, diminished GFR) are more pronounced in group SP than normotensive group WKY and hypertensive group SC without mesangial cell injury. While normotensive group WKY rats recovered completely from mesangial cell injury on day 28-42, glomeruli in group SP kept on persistent macrophage infiltration, $\alpha-S M A$ expression on day 42-56. In addition, glomerular capillary repair with the GECs was rarely seen in pronouncedly proliferative and sclerostic areas. The incidence of glomerular sclerosis and the level of proteinuria were markedly increased by day 56 in the group SP. Conclusions: Our results demonstrate that glomerular hypertension aggravate glomerular damage and glomerulosclerosis in this model of Thy 1 nephritis.
\end{abstract}




\section{Kidney Blood Pressure Research}

Wang/Kitamura/Shimizu/Yamanaka: Glomerular Damage

\section{Introduction}

Multiple pathogenetic mechanisms are considered to be responsible for the progression of renal diseases. Immunologically and hemodynamically mediated disturbance of glomerular filtration barrier are thought to be the major causes of end-stage renal failure [1]. Glomerular filtration barrier is constructed by the endothelial cells, the glomerular basement membrane (GBM) and the podocytes. Mesangial cells play a role in maintaining the three-dimensional structure of the capillary bed and normal glomerular function. Numerous evidence from human glomerulonephritis and experimental animal model indicate that initiating events targeting any of the four major components of the glomerulus can cause the destruction of glomerular architecture eventually leading to glomerular sclerosis [2]. It is well known that injection of anti-Thy I antibody into rats can cause severe mesangiolysis with capillary ballooning microaneurysm formation, and loss of endothelial cells, followed by a rebound mesangial cell proliferation and matrix synthesis, subsequently the most glomeruli spontaneously recover within 2-4 weeks [3-5]. However, there is so far no report evaluating the degree of glomerular damage in response to a single dose of anti-thy- 1 antibody and its functional consequences under glomerular hypertension. The stroke-prone spontaneously hypertensive rat strain (SHR-SP), which is established from the offspring of Wistar strain spontaneously hypertensive rat (SHR), provides best available animal analogue of hypertension in humans [6]. In addition, unilateral nephrectomy (UNX) in SHRs provokes increased glomerular capillary pressure compared to the normotensive same genetic strain UNX Wistar-Kyoto (WKY) rat resulting in further hemodynamically mediated renal injury [7]. In the present study, we investigated the course of renal damage of Thy- 1 nephritis under augmented situation of glomerular capillary hypertension using the SHR-SP with UNX model. Studies in rats with subtotal renal ablation have demonstrated that intraglomerular hypertension can cause endothelial cell injuries and initiate glomerular sclerosis [8]. Our experimental model not only has an immune-mediated mesangial injury, but also shows the severe and progressive process of glomerular damage due to glomerular capillary hypertension. Few studies have been carried out to elaborate the effects of hypertension on the natural course of Thy-1 nephritis [9] and none has been done to elucidate the influence of the endothelial cell damage in the process of proliferative glomerulonephritis under glomerular capillary hypertension. With our model, we aim to investigate hypertensive glomerular hemodynamic effects on the natural course of Thy-1 nephritis and to look into whether glomerular damage takes on pathogenetic relevance in the evolution of glomerular injury.

\section{Materials and Methods}

This study was carried out in strict accordance with the recommendations in the regulations for the Care and Use of Laboratory Animals of Nippon Medical School. The protocol was approved by Nippon Medical School Animal Ethics (Permit Number: 37-10). All surgery was performed under sodium pentobarbital anesthesia, and all efforts were made to minimize suffering.

Experimental design

Male 12-week-old SHR-SP rats and WKY rats (Sankyo Experimental Animal Supply, Japan) weighing between 240 340g, were used for in the study. 12-week-old SHR-SP rats were selected because the systemic hypertension had been developed by this age. Figure 1 shows the experimental design.

The animals received unilateral nephrectomy (left kidney) under ether anesthesia. After the operation, fifty-four SHR-SP were randomly divided into two groups: Group SP (experimental group of SHRSP) and Group SC (control group of SHR-SP). Group SP and Group WK (normotensive WKY) consisted of 42 rats for each group given a single intravenous injection of $60 \mu \mathrm{g}$ IgG/100g weight of monoclonal anti-thy1.1 antibody (OX-7; Cedarlane Laboratories, Toronto, Ont. Canada) through the tail vein. Seven rats were sacrificed on day 3, 7, 14, 28, 42, and 56, respectively. Group SC (control group of SHR-SP) consisted 


\section{Kidney \\ Blood Pressure Research}

Kidney Blood Press Res 2015;40:188-199

\begin{tabular}{l|l}
\hline DOI: $10.1159 / 000368494$ & (c) 2015 S. Karger AG, Basel
\end{tabular}

Published online: March 30, 2015

www.karger.com $/ \mathrm{kbr}$

Wang/Kitamura/Shimizu/Yamanaka: Glomerular Damage

Fig. 1. The in vivo experimental design. The line illustrates the time points of experiments after $O X 7$ administration (arrow) in group SP and $\mathrm{WK}$, and normal saline administration (in group SC) at 0 day after unnephrectomy. Numbers in parentheses represent the number of mice. Abbreviations: Un, unnephrectomy; IV, tail vein injection.

\begin{tabular}{|c|c|c|c|c|}
\hline Un SP SP & SP & SP & SP & SP \\
\hline OX7(7) (7) & (7) & (7) & (7) & (7) \\
\hline IV WK WK & WK & WK & WK & WK \\
\hline (7) (7) & (7) & (7) & (7) & (7) \\
\hline$\downarrow$ & & SC & $\mathrm{SC}$ & SC \\
\hline-1 & 1 & (5) & (5) & (5) \\
\hline $\begin{array}{lll}\mathrm{d} 0 & \mathrm{~d} 3 & \mathrm{~d} 7\end{array}$ & d14 & d28 & d42 & d56 \\
\hline
\end{tabular}

of 15 rats which only received normal saline in the same way and five rats were sacrificed on day 28,42 , and 56, respectively. Urine was collected at different times in metabolic cages where the rats had free access to water and standard rat chow. Twenty-four hour urine protein excretion was measured. Polyethylene catheter was inserted into the tail artery, and systolic blood pressure measurements were made by a Statham pressure transducer (P23) connected to a pressure recorder under light ether anesthesia. In addition, the blood sample was collected for serologic examination. The samples were measured by using an autoanalyzer (SRL, Tokyo, Japan).

\section{Morphological and immunohistological examination}

The right kidney was removed, fixed in $10 \%$ formalin or $4 \%$ paraformaldehyde, and embedded in paraffin. Sections were stained with hematoxylin-eosin (H\&E), periodic acid-Schiff (PAS) and periodic acid-methenamine silver (PAM) for light microscopic examination. Electron microscopical examination was performed in usual routine as described earlier [10].

For immunohistochemical examination, the following primary antibodies were included: a mouse anti-PCNA monoclonal antibody (Dako, glostrup, Denmark) for proliferating cell nuclear antigen (PCNA), an anti- $\alpha$-SMA antibody (abcam, Cambridge, MA, USA ) for smooth muscle actin (SMA), an anti-human type IV collagen antibody (Southeren Biotechnology, Birmingham, AL,USA), an mouse anti-rat ED-1 monoclonal antibody (IgG1, Chemicon (Millipore), CA, USA), and a polyclonal rabbit anti-rat thrombomodulin (TM) antibody (provided by Dr. David Stern, Columbia University, New York, N.Y., USA).

\section{Immunohistochemical double staining}

To detect proliferating endothelial cells, mesangial cells and phenotypic change of mesangial cells, sections were double immunostained for TM and PCNA, TM and ED-1 as well as $\alpha$-SMA and PCNA. Briefly, tissue sections were treated with $0.3 \%$ hydrogen peroxide in methanol for $30 \mathrm{~min}$ to block endogenous peroxidase activity, then incubated with avidin and biotin for 10 min each at room temperature (Biotin Blocking System; Dako), and incubated with $10 \%$ serum from the same species as the primary antibody source for $30 \mathrm{~min}$ at room temperature. The sections were first incubated with rabbit anti-TM or anti- $\alpha$-SMA antibody, respectively, and then incubated with biotinylated goat anti-rabbit IgG (Vector Laboratories), ABC/ HPR (Dako) for 45-60 min at room temperature, and color reaction was developed with 3,3'-diaminobenzidine tetrahydrochloride (DAB), and positive cells exhibited brown color for detecting proliferating endothelial cells (TM) and phenotypic change of mesangial cells ( $\alpha$-SMA), respectively. The sections were incubated with avidin and biotin for $10 \mathrm{~min}$ each at room temperature again, then were incubated with mouse anti-PCNA or anti-rat ED-1 antibody, followed by incubation with biotinylated horse anti-mouse IgG and avidin-biotin-peroxidase complex (ABC). The color reaction was developed using DAB substrate with Nickel. PCNA- and ED-1- positive cells exhibited gray-black nuclei for detecting proliferating cells and macrophages, respectively. Negative controls were processed in same way except for replacing each primary antibody with a non-immune IgG of the same species. Cross-reactivity between the two detection systems was checked by either omitting or substituting antibodies with PBS. Counter staining was with hematoxylin. Identification of Apoptotic cells was performed by TUNEL method according to the manufacturer's protocol (ApopTag Peroxidase In Situ Apoptosis Detection Kit S7100; Chemicon international, Temecula, CA, USA). 


\section{Kidney Blood Pressure Research}

Wang/Kitamura/Shimizu/Yamanaka: Glomerular Damage

\section{Immunolabeling for fluorescence microscopy}

The kidney tissues were fixed for $8 \mathrm{~h}$ at $4^{\circ} \mathrm{C}$ in $0.01 \mathrm{M}$ phosphate-buffer ( $\mathrm{pH} 7.4$ ) containing $4 \%$ paraformaldehyde. They were sequentially washed in 10, 15 and $20 \%$ sucrose in $0.001 \mathrm{M}$ phosphate-buffered saline (PBS; $\mathrm{pH} 7.4,4^{\circ} \mathrm{C}$ ) for 4 hours, respectively. And then were snap-frozen in O.C.T. embedding medium and stored at $-80^{\circ} \mathrm{C}$.

To accurately evaluate the numbers of GECs and glomerular capillary lumina, GECs were labeled using immunofluorescent probe. Briefly, the sections were incubated with a 1:10 diluted mouse monoclonal anti-rat endothelial cell antibody, RECA-1 (Sterotec Ltd., Oxford, United Kingdom) for 60 min at room temperature. After washing, the sections were incubated with FITC-conjugated goat anti-mouse IgG antibody for $60 \mathrm{~min}$ at room temperature. Nuclear counterstaining was made with 4, 6-diamidino-2-phenylindole (DAPI; Vector Laboratories, Inc., Burlingame, CA). Specimens were examined with a laser scanning confocal fluorescence microscopy (CLSM, TCS-SP; Leica Lasertechnik, Heidelberg, Germany) equipped with krypton/argon laser sources. Each field was checked using Nomarski optics. In this preparations, green fluorescence represented RECA-1 and nuclei showed a blue fluorescence. Images were collected using a Leitz $63 \times$ PL APO objective (numerical aperture NA =1.4). The means of 32 scans were obtained at a resolution of $512 \times 512$ pixels and analyzed using TCS-NT (Leica Laser-technik).

\section{Quantitative evaluation of histological findings}

In each kidney specimen, 60 100 glomerular cross sections were examined, counted, and averaged. The examination was performed in a blinded manner by two pathologists to measure the following variables at different time: 1) total cellularity: the mean number of nuclei per glomerular cross section in tissue sections stained with PAS; 2) total numbers of GECs and total numbers of glomerular capillary lumina: random fields at high magnitude on the cross section from each kidney were photographed, and their fluorescence signals were measured by Imager J software (available at http://rsbweb.nih.gov/ij/). The mean number of RECA-positive cells per glomerular cross section and the mean number of glomerular capillary lumina surrounded by RECA-positive cells per glomerular cross section were then calculated; 3 ) numbers of proliferating cells: the mean number of PCNA-positive cells per glomerular cross section; 4) the numbers of proliferating endothelial cells: the mean number of both TM-positive and PCNA-positive cells per glomerular cross section; 5) the numbers of proliferating mesangial cells: the mean number of both $\alpha$-SMA-positive and PCNA-positive cells per glomerular cross section; 6 ) the numbers of infiltrating macrophages: the mean number of ED-1 positive cells per glomerular cross section; 7) the percentage of glomeruli with ballooning lesions was evaluated for each kidney specimen; 8) the glomerular tuft area per glomerular cross section on H\&E-staining with a digital camera were photographed, and the images were calculated by the Imager-Pro Plus (Media Cybernetics, Silver Spring, MD) software.

Semiquantitative evaluation of histological findings.

A minimum of 60 glomeruli (range, 60-100) in each kidney specimen was examined. A semiquantitative score was graded from 0 to $4+$ according to the percentage of glomerular involvement in a blinded manner by two pathologists using modified method of Floege et al. [11] and Thomas SE, et al. [12] The grade score was set as $0=0 \%, 1+=1-25 \%, 2+=26-50 \%, 3+=51-75 \%$ and $4+=76-100 \%$. The mean score per glomerular cross section was calculated for the following variables at various time intervals: 1) phenotypic change of mesangial cells: the positive area of immunoperoxidase stains for $\alpha$-SMA per glomerular cross section; 2) the extent of mesangial matrix expansion: the positive area of immunoperoxidase stains for type IV collagen per glomerular cross section; 3) glomerulosclerosis: segmental or global collapse or obliteration of glomerular capillary lumina associated with increased glomerular matrix and adhesion of the tuft to Bowman's capsule were defined as glomerulosclerosis. The degree of sclerosis in each glomerulus was evaluated by the presence of increased amounts of periodic acid-methenamine silver (PAM) positive material per glomerular cross section.

\section{Statistical analysis}

All values are expressed as mean \pm standard deviation (SD) for individual groups. Comparison between two groups was made by the unpaired Student's t test. Statistical significance was defined as $\mathrm{P}<0.05$. 


\section{Kidney \\ Blood Pressure Research}

\section{Results}

Hemodynamics proteinuria and renal function measurements

Figure 2A shows that the systolic blood pressures in both groups SP and SC before and throughout the study were higher than the age-matched normotensive group WK at the age of week 12. However, no significant difference was observed between the group SP and the group SC. As hypertension progresses, the level of urinary protein excretion between two group is different, proteinuria was more severe in the group SP than in the group SC $(\mathrm{P}<0.01)$ (Figure 2B). Correspondingly, significantly elevated levels of blood urea nitrogen (BUN) and serum creatinine $(\mathrm{Cr})$ were noted by day 56 in the group SP $(\mathrm{P}<0.01)$ (Figure $2 \mathrm{C}-\mathrm{D})$, indicating substantial renal damage after Thy-1 nephritis.

Morphological changes of the glomerulus

Markedly diminished glomerular cellularity was noted on day 3 after antibody administration in both groups SP and WK, compared to their baseline $(\mathrm{P}<0.01)$ (Figure $3 \mathrm{~A})$. Loss of glomerular cellularity coincides with loss of mesangial cells, marking diffusive mesangial cell lysis. Mesangial proliferation eventually dominated and reached its peak on day 14 and most of the glomeruli have recovered to their original structure on day 28 in WK rats. In contrast, SP rat showed a profound lesion and slow onset mesangial proliferation, and persistent lesions were presented in the majority of glomeruli throughout the study (Figure 3A). Similar pattern can be seen based on

Fig. 3. Cellular change at different time points. The number of total cells (A), proliferating cell nuclear antigen (PCNA) positive cells (B) per glomerular cross section during the course of the experiment. Values are expressed as mean $\pm \mathrm{SD}$. ${ }^{*} \mathrm{P}<0.05 ;{ }^{*} \mathrm{P}<0.01$ in the group SP versus group WK and group SC, respectively.

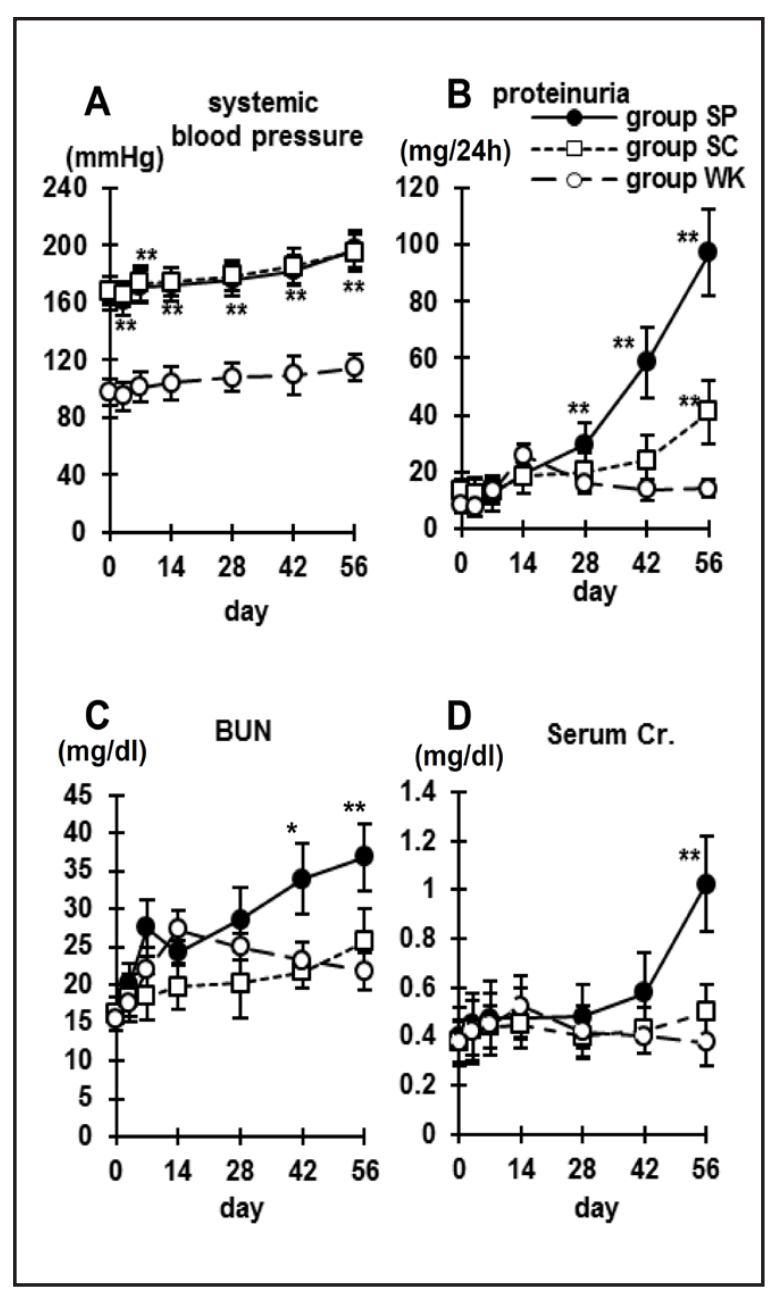

Fig. 2. Changes of systemic blood pressure (A), urinary protein (B), blood urea nitrogen (BUN) (C) and serum creatinine (Cr) (D) in parameters during the course of the experiment. Values are expressed as mean \pm SD. ${ }^{*} \mathrm{P}<0.05$; ${ }^{*} \mathrm{P}<0.01$ in the group $\mathrm{SP}$ versus group $\mathrm{WK}$ and group $\mathrm{SC}$, respectively.

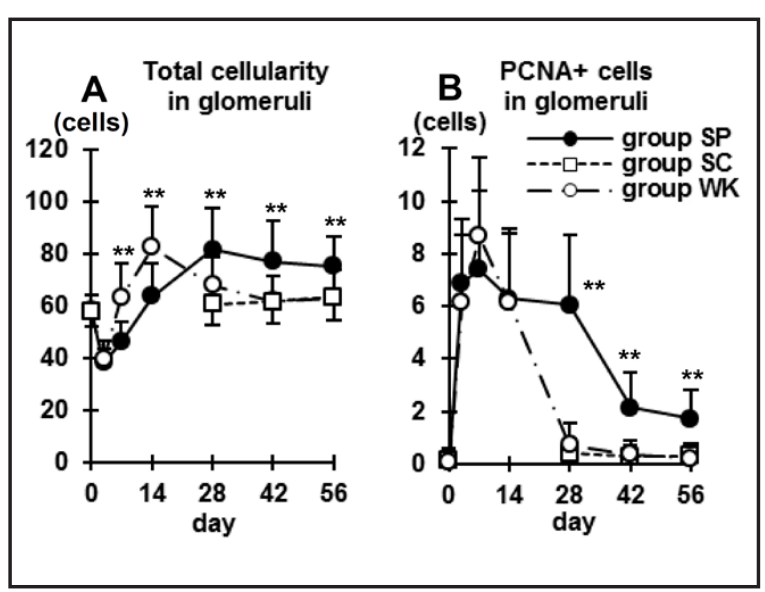




\section{Kidney \\ Blood Pressure Research}

Kidney Blood Press Res 2015;40:188-199

\begin{tabular}{l|l}
\hline DOI: 10.1159/000368494 & C 2015 S. Karger AG, Basel
\end{tabular}

Published online: March 30, 2015

www.karger.com/kbr

193 proliferative cells, as shown by PCNA staining: there was still active cell proliferation in glomerulus in SP rats while complete recovery in WK rat has been achieved (Figure 3B). The impaired glomerular capillary network was evident, as measured by quantitative segmental microaneurysmal balloonings (Figure 4A) and glomerular sclerosis by counting PAM staining (Figure 4B-D). Balloonings were mainly observed on day 7-14 in the group WK, and on day 28-42 in the group SP. Hypercellularity, matrix expansion and $\alpha$-SMA expression peaked on day 14 and gradually subsided in the group WK (Figure 3A-B and 5A-D). However, persistent $\alpha$-SMA expression was still observed at the later phase in the group SP and no $\alpha$-SMA expression was observed in mesangial area in the group SC. The change of mesangial proliferation in both groups SP and WK is in consistence to the change of PCNA+ cells (Figure 5A-D). In SP rats, although some glomeruli spontaneously recovered to normal structure within 42-56 days, a greater proportion of glomeruli still displayed pronounced proliferative changes, proliferating PCNA+ cells showed persistent $\alpha$-SMA expression, referring to a big difference between SP and WK rats, the change pattern of $\alpha$-SMA and PCNA is very similar.

In order to assess glomerular sclerosis, Type IV collagen antibody was used to stain extracellular matrix, and PAM staining (Figure 6A-C) was also applied. In SP rats, heavily deposit of type IV collagen in glomerulus in SP rats is very common. Progressive accumulation of type IV collagen was detected during days 28-56 after Thy1.1 administration. Progressive glomerular sclerosis was further demonstrated in PAM staining and collapse of the glome-

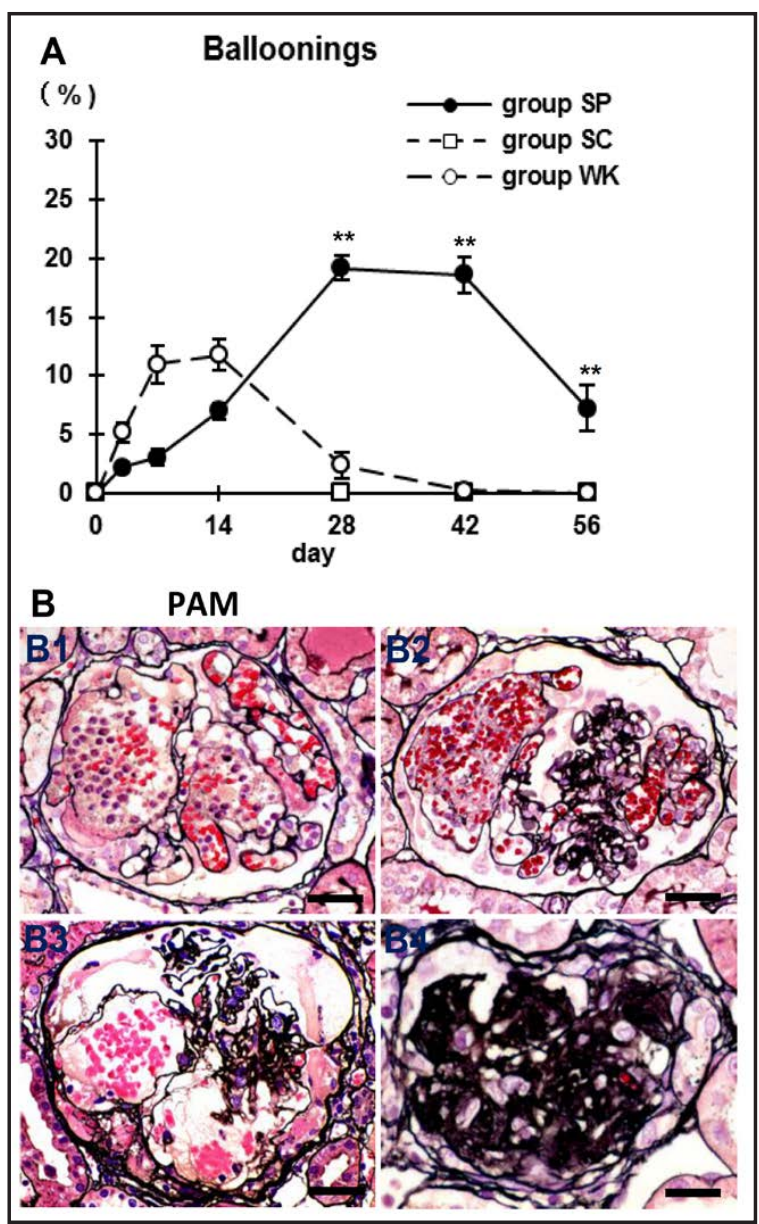

Fig. 4. The changes of mesangiolytic microaneurysmal ballooning in Thy-1 nephritis. The number of total balloonings (A). Values are expressed as mean \pm SD. ${ }^{*} \mathrm{P}<0.05 ;{ }^{*} \mathrm{P}<0.01$ in the group $\mathrm{SP}$ versus group WK and/or group SC. Persistent damage of glomerular capillary network in SHR-SP with Thy-1 nephritis. Mesangiolytic microaneurysmal ballooning as the severest form of mesangiolysis (B) is shown on day 14 (B1). Ballooning lesions or failure of re-establishing the glomerular architecture with focal and segmental glomerular sclerosis are observed on day 28 (B2) and 42 (B3). Glomerular sclerosis is observed on day 56 (B4). All bars $=30 \mu \mathrm{m}$.

rulus, loss of glomerular capillary network, and intensive deposit extracelluar matrix were often observed at the later phase in group SP. Mild deposit of type IV collagen in the group SC and group WK rats on day 56. When type IV collagen deposit was quantified, peak deposit of collagen IV in WK rats occurred on day 14 after Thy1.1 administration, followed by steady drop, the pattern coinciding with cell proliferation. The increase of kidney weight and glomerular volume was observed following uninephrectomy. There are no significances between each group (Figure 7).

Infiltration of macrophages was observed in impaired glomeruli in $\mathrm{SP}$ and $\mathrm{WK}$ rats ( $\mathrm{Fi}$ gure $8 \mathrm{~A}$ ). Although accumulation of ED-1-positive macrophages in SP rats with nephritis 


\section{Kidney \\ Blood Pressure Research}

Kidney Blood Press Res 2015;40:188-199

\begin{tabular}{l|l}
\hline DOI: 10.1159/000368494 & C 2015 S. Karger AG, Basel
\end{tabular}

Published online: March 30, 2015

www.karger.com/kbr

194

Fig. 5. Proliferation of activated mesangial cells in a damaged glomerulus. Glomerular immunohistochemical staining scores for $\alpha$-SMA (A) and double staining for $\alpha$-SMA and PCNA (B) per glomerular cross section in the different groups. Values are expressed as mean $\pm \mathrm{SD}$. ${ }^{*} \mathrm{P}<0.05 ;{ }^{* *} \mathrm{P}<0.01$ in the group SP versus group WK and group SC, respectively. Two pairs of serial sections with immunostaining for $\alpha$-SMA and PAM stain (C) and double immunostaining for $\alpha$-SMA and PCNA and PAM stain (D). Persistent proliferation of activated mesangial cells by immunohistochemical staining for $\alpha$-SMA was observed in glomerulus of group SP at day 56 after Thy-1 nephritis induction (C1). The expression of $\alpha$-SMA was only observed in glomerular arterioles in the group SC at day 56 without Thy-1 nephritis (C2). D1: One week after disease induction in the group SP. Some glomerular cells begin to proliferate and double immunostaining for PCNA and $\alpha$-SMA shows the mesangial cell origin of PCNA positive proliferating cells $(\downarrow)$. D2: Six weeks after disease induction in the group SP. Mesangial cell proliferation with glomerular capillary reconstruction is seen in the markedly destructive glomerular tuft $(*)$. Double-labeled PCNA $+/ \alpha-S M A+$ proliferating and activated mesangial cells $(\downarrow)$ are identified in the same glomerulus. All bars $=30 \mu \mathrm{m}$.

was predominantly located in mesangial areas, ED-1 positive macrophages were also found along with endothelium by double labeling (Figure 8B-C). In a time course, early infiltration of macrophages was observed on day 3 in both groups of SP and WK rats. Macrophage infiltration in SP rat with nephritis was quite persistent (Figure 8A-C), whereas filtration of macrophages eventually resolved in WK

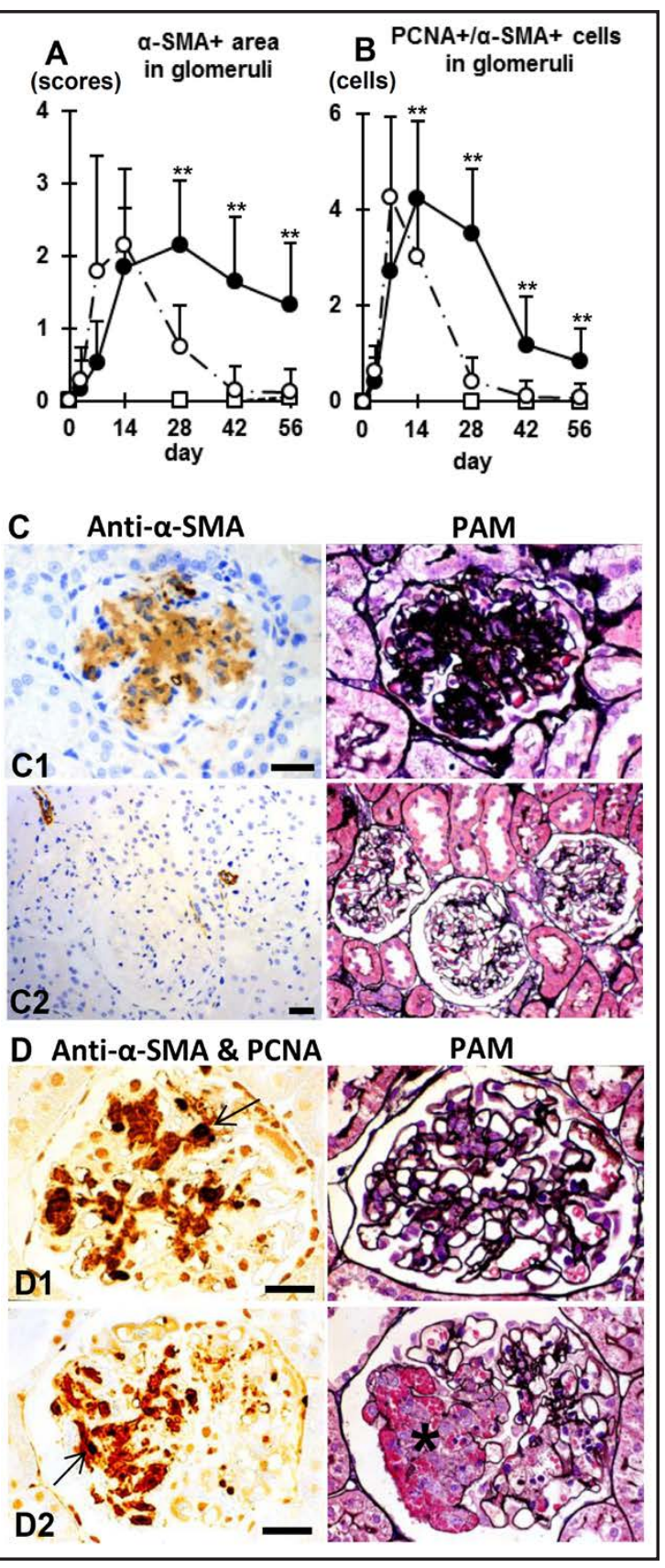
rats. TUNEL staining revealed that a persistent increase in the number of apoptotic cells in glomeruli in the group SP compared to in the group SC and WK (data not show).

Changes of glomerular endothelial cells (GECs) and capillary repair

A reduction of RECA-1-positive GECs (Figure 9) and a subsequent increase of proliferative GECs (PCNA+/TM+) (Figure 10) were observed on day 3 after Thy-1.1 antibody induction in both groups SP and WK compared to their baseline $(\mathrm{P}<0.01)$, demonstrating that GEC damage followed the acute mesangial injury. RECA-1-positive and TM-positive GECs or glomerular capillary lumina were lost in segmental microaneurysmal ballooning lesions (Fig. 9-10), but a few GECs still remained on the periphery. Glomerular capillary repair was achieved through proliferation of remaining viable endothelial cells. Numerous proliferating endothelial cells (PCNA+/TM+) were found in mesangiolytic and ballooning 


\section{Kidney Blood Pressure Research}

Wang/Kitamura/Shimizu/Yamanaka: Glomerular Damage

Fig. 6. Accumulation of type IV collagen and glomerulosclerosis. Glomerular immunohistochemical staining scores for type IV collagen (A) and the score of glomerulosclerosis (B) per glomerular cross section. Values are expressed as mean \pm SD. ${ }^{*} \mathrm{P}<0.05$; ${ }^{* *} \mathrm{P}<0.01$ in the group SP versus group $\mathrm{WK}$ and group $\mathrm{SC}$, respectively. Two pairs of serial sections with immunostaining for type IV collagen and PAM stain (C). C1: The eight week after disease induction in group-SP. Marked proliferative lesions, focal and segmental glomerulosclerosis and extensive expression of the type IV collagen are noted in the group SP. Most of the glomeruli reveal the normal structure and no evidently detectable levels of glomerular type IV collagen expression in the group SC (C2) and WKY (C3) by day 56 . All bars $=60 \mu \mathrm{m}$.

lesions, and the newly capillary network gradually developed accompanied by mesangial cell proliferation and matrix accumulation. Active capillary repair was obvious at day 7 (Fig. 10A). Most of RECA-1-positive glomerular capillary lumina revealed a strong resemblance to their original structure at days 2856 in WK. However, persistent damage of the glomerular capillary network, failure of reconstruction of the glomerular architecture and progression to glomerulosclerosis

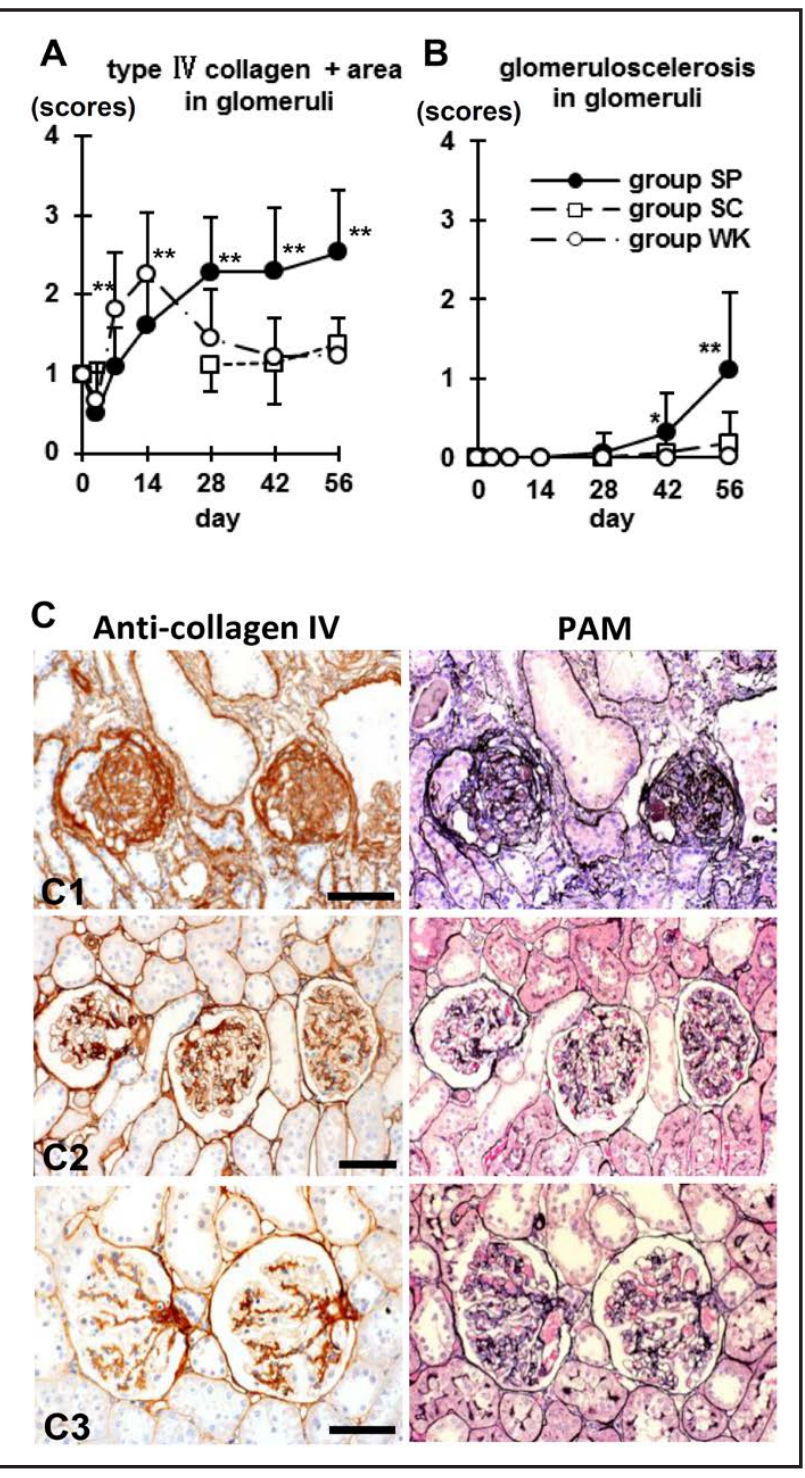

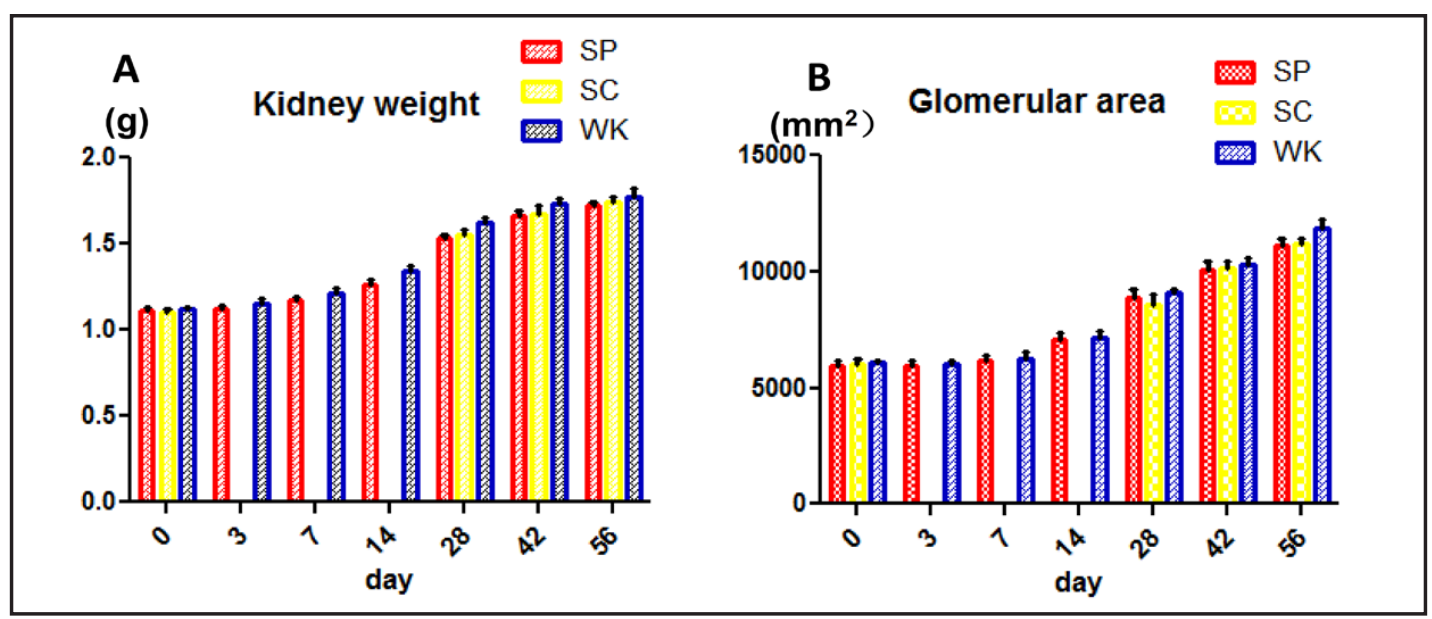

Fig. 7. Kidney weights and morphometry results and time-course following uninephrectomy. The gradual increase in kidney weight and glomerular size as time-course compared to their early phase. There was no stastistically significant between each group at same timepoint. 


\section{Kidney Blood Pressure Research}
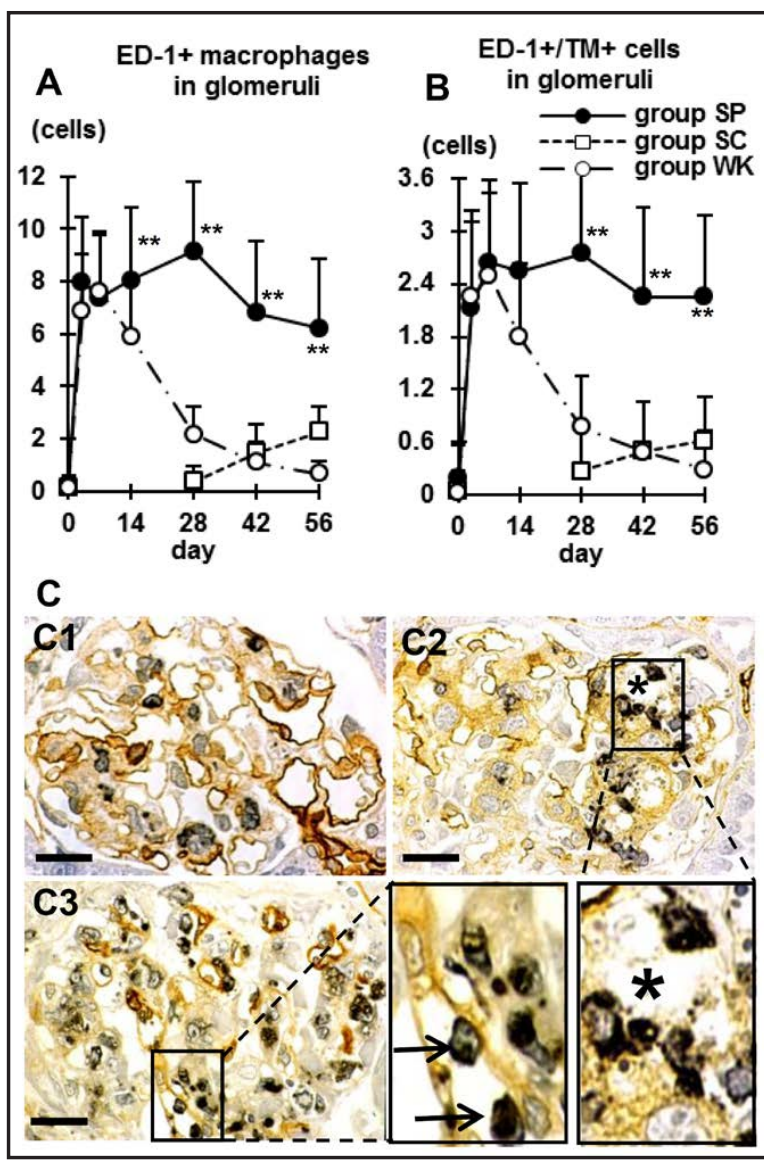

Fig. 8. Infiltration of macrophages. Glomerular immunohistochemical staining scores for ED1 (A) and double staining for TM and ED1 (B) per glomerular cross section. Persistent pronounced infiltration of macrophages in damaged glomeruli is noted in SHR-SP with Thy-1 nephritis. Values are expressed as mean $\pm \mathrm{SD}$. ${ }^{*} \mathrm{P}<0.05$; ${ }^{* *} \mathrm{P}<0.01$ in the group SP versus group $\mathrm{WK}$ and group $\mathrm{SC}$, respectively. Although accumulation of ED-1-positive macrophages is predominantly noted in mesangial areas (C1), numerous infiltrating macrophages are also found in areas where glomerular endothelial cell injuries $\left(^{*}\right)$ (C2) and periphery of markedly mesangial proliferation and glomerular sclerosis ( $\downarrow$ ) (C3). (C1: 14 days, C2: 28 days, C3: 56 days) (double immunostaining with ED-1 (dark-blue) and TM (brown). All bars $=30 \mu \mathrm{m}$.

was observed in the group SP (Figure 4, 6 and 10). Endothelial cells were extensively detached or desquamated from the glomerular basement membrane (GBM) (Figure 11), and the mesangium were observed with increased influx of blood components into the ballooning lesions and mesangial areas (Figure 4B and 11). The capillary repair with involvement of endothelial cells was absent or rare in markedly proliferative and sclerotic areas, but proliferative GECs were still presented on the periphery of

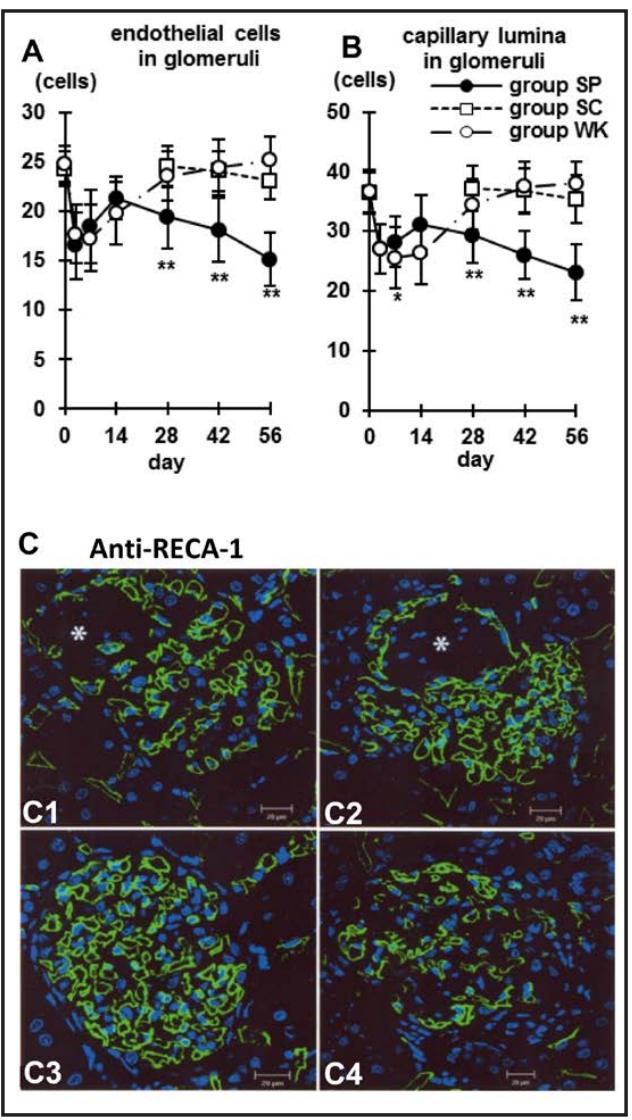

Fig. 9. Changes of glomerular endothelial cells. The number of glomerular endothelial cells (A), glomerular capillary lumina (B) staining for RECA-1 (green) by confocal fluorescent microscopy. Values are expressed as mean $\pm \mathrm{SD} .{ }^{*} \mathrm{P}<0.05 ;{ }^{* *} \mathrm{P}<0.01$ in the group $\mathrm{SP}$ versus group $\mathrm{WK}$ and group $\mathrm{SC}$, respectively. Fluorescent confocal image of RECA-1 in GECs and peritubular capillaries (PTC) shows green linear fluorescence with endothelial cell nucli (blue) (DAPI-stained nuclei) (C) along the glomerular capillary lumina and PTC lumina(C). The plasma membrane of GECs and PTC endothelial cells is exclusively reactive for RECA-1. The lack of luminal staining in areas of ballooning lesion $\left({ }^{*}\right)$ was shown in the group-SP on day 14 (C1) and 28 (C2), the glomerular capillary reconstruction with increase in RECA-1 expression in the damaged glomerulus on day 42 (C3), and notable reduce of RECA- 1 + glomerular capillary lumina in markedly proliferative and sclerotic glomerulus on day 56 (C4). All bars $=20 \mu \mathrm{m}$. 


\section{Kidney Blood Pressure Research}

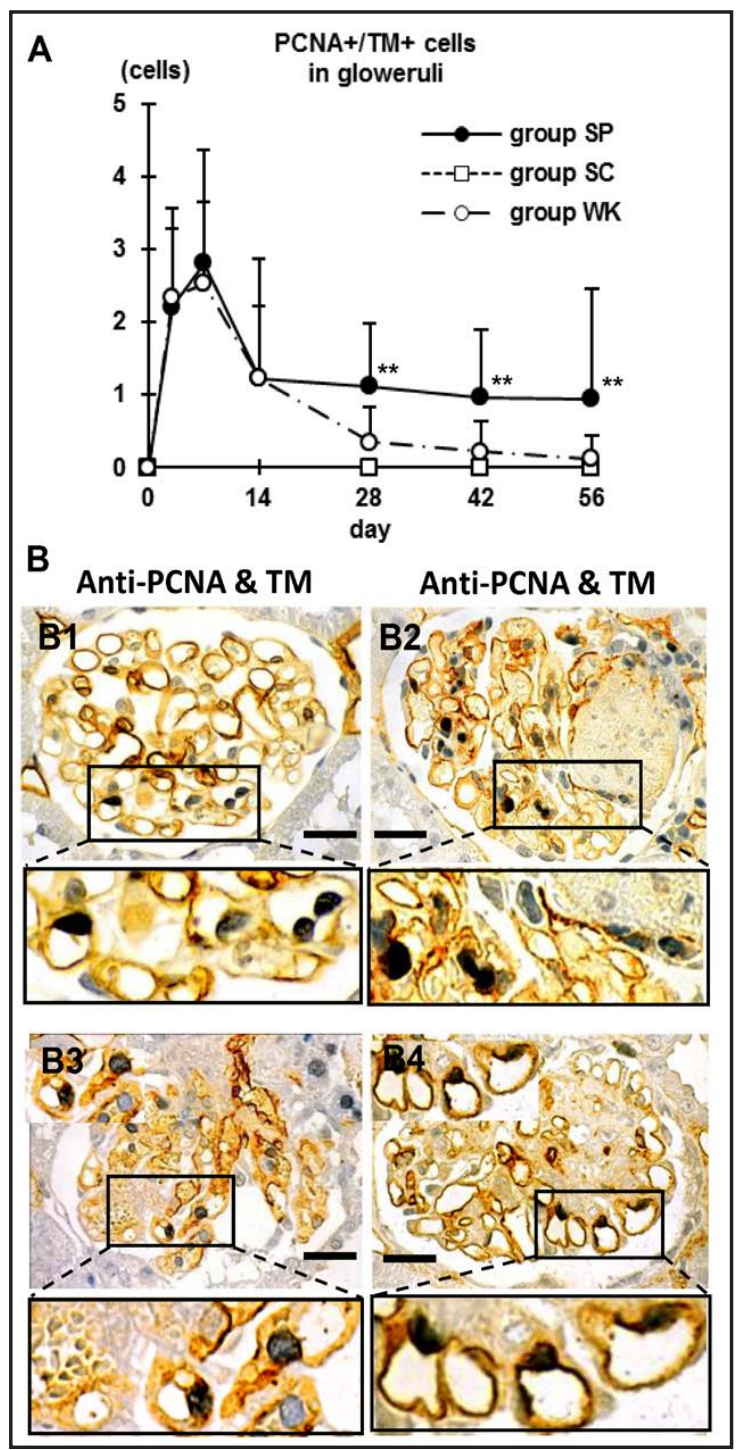

Fig. 10. The change of endothelial cell proliferation. The number of proliferating glomerular endothelial cells (PCNA+/ $\mathrm{TM}+$ ) (A) per glomerular cross section. Values are expressed as mean $\pm \mathrm{SD}$. ${ }^{*} \mathrm{P}<0.05$; ${ }^{* *} \mathrm{P}<0.01$ in the group SP versus group SC and/or group WK. B: double immunostaining with PCNA (dark-blue) and TM (brown) in the group SP. Mesangial cell-lysis with many PCNA+/TM+ proliferating GECs was observed in the damaged glomerulus on day 3 (B1). The severely damaged capillary network with loss of GECs in ballooning lesions and capillary repair with GEC proliferation was noted on day 28 (B2). Capillary growth with endothelial cell proliferation was found from the glomerular vascular pole extending to the periphery (B3). The rare or absent capillary repair with GECs was noted in pronouncedly proliferative and sclerostic areas and compensatory capillary regeneration with GEC proliferation was found in the periphery of severely damaged regions of glomerulus (B4). All bars $=30 \mu \mathrm{m}$.

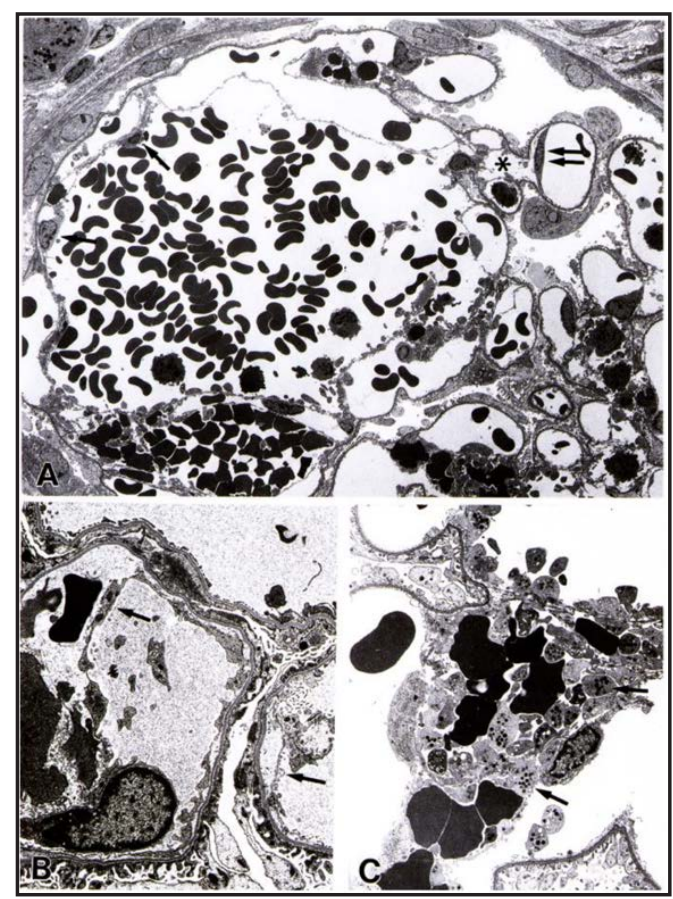

Fig. 11. Electron micrographs of the glomerulus in group SP on day 42. A: capillary loops lose their original structure, forming new microaneurysmal ballooning, but a small number of endothelial cells $(\downarrow)$ still remain in the periphery $(\times 500)$. The endothelial cell $(\downarrow \downarrow)$ with loss of supporting function of mesangium due to mesangiolysis $\left({ }^{*}\right)$ is remodeled in shape and is markedly elongated to attach the GBM (B): The desquamation of endothelial cells $(\downarrow)$ from GBM $(\times 3000)$. C: platelets in the mesangial area of markedly damaged glomeruli $(\times 2000)$.

severely damaged regions of the glomerulus (Fig. 10B).

\section{Discussion}

An interesting feature of this study is that the mesangiolytic microaneurysmal ballooning, which is the severest form of mesangiolysis, was still seen in the later phase of Thy-1 nephritis in the group SP. The mechanisms responsible for phenomenon are not fully elucidated in the present study, intraglomerular hypertension, an increase in the number of apoptotic 


\section{Kidney Blood Pressure Research}

cell and inflammatory cell infiltration could be responsible. First, it could be caused by the fact that Thy- 1 nephritis in our study was developed in the context of hypertension. When the glomerular filtration rate and the glomerular capillary pressure are increased persistently by hypertension, increased mechanical tension may be the contributing factor in the destruction of capillary networks and persistence of microaneurysmal ballooning through the study, hence the failure of vascular remodeling in the group SP.

In the present study, we found that GECs gradually reduced or disappeared in the group SP, and capillary repair was rarely seen in severely damaged glomeruli, resulting in angiogenesis being prevented, with subsequent sclerosis taking place in the impaired region. These results implicate that glomerular endothelial cell injury exerts significant influences on the progression and repair process of glomerular disease and impaired capillary repair with the absence of the development of the capillary network accounts for the pathogenesis of the cause and progression of glomerular sclerosis associated with proteinuria and loss of glomerular function.

In the present study, the kidney of the group SP still exhibited markedly proliferative changes which remained an evident expression for $\alpha$-SMA and proliferating and activated mesangial cells and markedly increased type IV collagen deposition in the damaged glomerulus during the later stage after Thy- 1 nephritis induction. This indicates that persistent proliferative activity is responsible for progressive glomerular injury in the group SP.

A persistent infiltration of macrophages was observed in damaged glomeruli in rats of the group SP. Macrophage recruitment is not only observed predominantly in the mesangial area, but was also also found in the endothelial cells of glomerular capillary. The endothelium is the side of initial contact with blood leukocytes during inflammation, thus the endothelium may be functionally adapted to serve as an early and sustained source of chemokines that recruits specific leukocytes such as macrophages to the site of inflammation. As shown in Figure 6, numerous infiltrating macrophages were found in the areas where GEC injury was evident, suggesting the involvement of macrophages in GEC injury and subsequent destruction of the glomerular capillary structure. This indicates that accumulation of macrophages contributes to the progression of glomerular damage occurring in the experimental proliferative glomerulonephritis in UNX SHR-SP.

Patients with chronic renal disease are often associated with a loss of total nephron mass. Progressive renal injury to chronic renal insufficiency in these patients with clinically apparent reduction in functioning nephron number can be accelerated under uncontrolled systemic hypertension. In the present study, substantial proteinuria and significantly elevated levels of blood BUN and Cr were noted in the group SP indicating widespread glomerular injury and glomerulosclerosis after Thy-1 nephritis were associated with increased glomerular capillary pressures.

The finding in the present study provides a good explanation for these clinical observations in all hypertensive patients with renal functional abnormalities due to progressive glomerular damage and sclerosis following the initial immune-mediated challenge to the glomerulus.

\section{Conclusion}

The present study confirms that the glomerular damage of Thy-1 nephritis is aggravated by concomitant glomerular hypertension and that the macrophage infiltration mediates further glomerular lesions in the kidney exposed to systemic and intraglomerular hypertension. This indicates that glomerular damage takes on pathogenetic relevance as a major factor in progression of glomerular injury to glomerular sclerosis.

\section{Abbreviations}

$\mathrm{ABC}$, avidin-biotin-peroxidase complex; BUN, blood urea nitrogen; CFM, confocal fluorescence microscopy; GBM, glomerular basement membrane; GFR, glomerular filtra- 


\section{Kidney \\ Blood Pressure Research}

tion rate; H\&E, hematoxylin-eosin; PAM, periodic acid-methenamine silver; PAS, periodic acid-Schiff; PBS, phosphate-buffered saline; PCNA, proliferating cell nuclear antigen; SHR, spontaneously hypertensive rat; SHR-SP, the stroke-prone spontaneously hypertensive rat strain; SMA, smooth muscle actin; TM, thrombomodulin; UNX, unilateral nephrectomy; WKY, Wistar-Kyoto.

\section{Disclosure Statement}

The authors of this manuscript state that they do not have any conflict of interests and nothing to disclose.

\section{Acknowledgments}

This research was supported by Nippon Medical School and Shandong University. The authors are grateful to Drs. K. Hiroshi, M. Yukinari, E. Jin, T. Mori and Mr. T. Arai for their advice and technical assistance and Drs. D. Stern and Y. Yuzawa for providing the anti-TM antibody.

\section{References}

1 Bergijk EC, Heer ED, Hoedemaeker PJ, Bruijn JA: A reappraisal of immune-mediated glomerulosclerosis. Kidney Int 1996;49:605-611.

2 Pozzi A, Zent R: TGF- $\beta$ sequestration by mesangial cell integrin $\alpha v \beta 8$ : A novel mechanism of the destruction of glomerular architecture glomerular endothelial cell regulation. Am J Pathol 2011;178:485489.

3 Ishizaki M, Masuda Y, Fukuda Y, Sugisaki Y, Yamanaka N, Masugi Y: Experimental mesangioproliferative glomerulonephritis in rats induced by intravenous administration of anti-thymocyte serum. Acta Pathol Jpn 1986;36:1191-1203.

4 Bagchus WM, Hoedemaeker PJ, Rozing J, Bakker WW: Glomerulonephritis induced by monoclonal anti-Thy 1.1 antibodies. A sequential histological and ultrastructural study in the rat. Lab Invest 1986;55:680-687.

5 Iruela-Arispe L, Gordon K, Hugo C, Duijvestijn AM, Claffey KP, Reilly M, Couser WG, Alpers CE, Johnson RJ: Participation of glomerular endothelial cells in the capillary repair of glomerulonephritis. Am J Pathol 1995;147: 1715-1727.

6 Okamoto K, Hazama F, Yamori Y, Haebara H, Nagaoka A: Pathogenesis and prevention of stroke in spontaneously hypertensive rats. Clin Sci Mol Med Suppl 1975;2:161-163.

7 Dworkin LD, Feiner HD: Glomerular injury in uninephrectomized spontaneously hypertensive rats. A consequence of glomerular capillary hypertension. J Clin Invest 1986;77:797-809.

8 Lee LK, Meyer TW, Pollock AS, Lovett DH: Endothelial cell injury initiates glomerular sclerosis in the rat remnant kidney. J Clin Invest 1995;96:953-964.

9 Wenzel UO, Wolf G, Thaiss F, Helmchen U, Stahl RA: Renovascular hypertension dose not influence repair of glomerular lesions induced by anti- thymocyte glomerulonephritis. Kidney Int 2000;58:1135-1147.

10 Ohashi R, Shimizu A, Masuda Y, Kitamura H, Ishizaki M, Sugisaki Y, Yamanaka N: Peritubular capillary regression during the progression of experimental obstructive nephropathy. J Am Soc Nephrol 2002;13:1795-1805.

11 Floege J, Johnson RJ, Gordon K, Iida H, Pritzl P, Yoshimura A, Campbell C, Alpers CE, Couser WG: Increased synthesis of extracellular matrix in mesangial proliferative nephritis. Kidney Int 1991;40:477488.

12 Thomas SE, Anderson S, Gordon KL, Oyama TT, Shankland SJ, Johnson RJ: Tubulointerstitial disease in aging: evidence for underlying peritubular capillary damage, a potential role for renal ischemia. J Am Soc Nephrol 1998;9:231-242. 